Escuela de Ciencias Sociales y Humanidades, UNED, C.R.

URL: http://investiga.uned.ac.cr/revistas/index.php/espiga/index

ISSN: 1409-4002 • e-ISSN: 2215-454X

Doi: http://dx.doi.org/10.22458/re.v17i35.1819

\title{
Interrogar el propósito de las ciencias sociales ${ }^{1}$
}

\author{
Diego Chaverri-Chaves* \\ iD https://orcid.org/0000-0003-0708-4289
}

Recibido: 27 de junio, 2017 - Aceptado: 29 de mayo, 2018

\section{RESUMEN}

Este artículo presenta varios datos sobre el contexto de las ciencias a nivel global y regional, los cuales permiten posteriormente una discusión sobre el efecto decisivo de las condiciones sociales sobre las disciplinas científicas que implican desigualdades internas. Este trabajo se nutre de fuentes documentales e información reciente y pertinente; aunado a un posicionamiento teórico crítico de la ciencia, según el cual, se sostiene que la sociedad define constantemente la función y utilidad de las ciencias sociales, señalando así el aprendizaje mutuo entre ciencias sociales y ciencias naturales.

Palabras clave: ciencia, ciencias sociales, conocimiento, desigualdad.

Formato de citación según APA

Chaverri-Chaves, D. (2018). Interrogar el propósito de las ciencias sociales. Revista Espiga 17(35), 63-76. Doi: http://dx.doi.org/10.22458/re.v17i35.1819

Formato de citación según Chicago-Deusto

Chaverri-Chaves, Diego. «Interrogar el propósito de las ciencias sociales». Revista Espiga 17, n. $^{\circ} 35$ (junio, 2018): 63-76. Doi: http://dx.doi.org/10.22458/re.v17i35.1819

* Académico de la Escuela de Sociología de la Universidad Nacional, Costa Rica. Licenciado y máster en Sociología por la Universidad de Costa Rica, con estudios de grado en Filosofía por la misma universidad. Colabora también con la UCR y la UNED en cursos y trabajos finales de graduación de grado y posgrado. Docente e investigador, Costa Rica. Correo: saludsociologia@gmail.com

1. Este artículo es parte del trabajo del autor durante los últimos cuatro años en la Escuela de Sociología de la Universidad Nacional de Costa Rica (UNA) sobre sociología del conocimiento, el cual se expuso parcialmente en la conferencia inaugural de la Primera Jornada de Investigación en Ciencias Sociales y Humanidades, el 03 de mayo de 2016, en la Universidad Estatal a Distancia (UNED). 


\section{Introducción: Las ciencias también como problema científico}

Metodológicamente, este artículo emerge de un trabajo de investigación documental sobre ciencia y sociedad, con revisión de fuentes de información locales e internacionales, por lo cual es necesario advertir que indudablemente la cantidad de datos disponible sobre esta materia es amplio, heterogéneo metodológicamente y en aumento; esto en parte es suscitado por el interés creciente en las ciencias desde la segunda mitad del siglo XX, a partir de la mayor inversión del mercado en la innovación tecnológica y la investigación asociadas al desarrollo económico de los capitales².

En consecuencia, este artículo se enfoca en fuentes más recientes y prioriza dentro de la información disponible a aquellos datos que muestran rasgos sociales de las ciencias en el siglo XXI, con lo cual no se pretende agotar la discusión, sino aproximarla a la tesis que sostiene que dichos rasgos son importantes para entender las condiciones sociales en la dinámica de desarrollo de las ciencias. Se observan entonces reportes sobre el desempeño de la ciencia en el mundo actual, que incluyen información sobre la percepción de la ciencia, su producción de información, la cantidad de personas y horas de trabajo científico, los presupuestos de inversión en investigación y desarrollo, así como las fuentes de ese financiamiento.

Para el enfoque teórico, se ofrece un abordaje sociológico crítico que señala el condicionamiento social paradójico de las ciencias, desarrollado por el sociólogo Norbert Elias, quien plantea que las crecientes dinámicas sociales de especialización del trabajo en la investigación explican en buena medida el avance de la ciencia; pero, esto genera que la ciencia en su desarrollo histórico reciente sea cada vez más dependiente de sus condiciones sociales ${ }^{3}$, esto es lo que se expone con la información recabada, pero manteniendo un diálogo con otros autores relevantes en filosofía de la ciencia, epistemología, sociología del conocimiento y campos afines, para la explicación de este fenómeno.

Las ciencias, entendidas simultáneamente como acopio de conocimiento y como actividad investigativa, han venido logrando, por lo menos durante los últimos tres siglos, mayores conocimientos objetivos de la realidad, son formas de conocimiento no incondicionadas, con el potencial de mostrar importantes facetas de los alcances y las limitaciones de las capacidades cognoscitivas de la especie humana, y es en buena medida cuestionando e indagando sobre sus condiciones y características que se pueden superar sus fronteras. Además, el cuestionamiento de las ciencias ha construido a estas en objeto de su propio oficio; es decir, hay ciencia de la ciencia, como se verá en el desenvolvimiento de este texto.

Las ciencias pueden tener beneficios culturales, aumentado o precisando el conocimiento, evacuando perplejidades, puede reportar ventajas económicas al aplicar conocimientos en la producción o comercialización de bienes y servicios o puede suponer mejoras tecnológicas en todo un amplio repertorio de aparatos; sin embargo, interrogar la razón de ser de las ciencias tiene inherentemente un contenido existencial que supera

2. Ernest Mandel, Capitalismo tardio (México: Ediciones ERA, 1979).

3. Norbert Elias, Compromiso y distanciamiento (España: Ediciones Península, 2002). 
estas primeras respuestas más pensadas para las ciencias aplicadas y su beneficio utilitario o desarrollista, pues toda la actividad científica requiere la vida humana, pero tal vida no se agota en la ciencia que es una de sus formas de experiencia y manifestación, no la única. Este problema filosófico no debe suponer un desvío obligatorio a asuntos deontológicos de las ciencias; por el contrario, este breve artículo se dedica a señalar condiciones sociales fácticas de las ciencias y las relaciones que sostienen entre sí para puntualizar algunos importantes efectos probables sobre su avance, su retroceso o su estancamiento. Si bien el énfasis en esta primera aproximación se pondrá en ciencias sociales, es prudente advertir que finalmente la división entre ciencias (cualesquiera de estas) supone un arbitrio humano y es respuesta a la división social del trabajo, más o menos justificado metódica o técnicamente, pero la lógica del sujeto humano, cognoscente, es capaz de entrelazar los problemas de todas las disciplinas ${ }^{4}$, a pesar de lo cual hay consecuencias de hecho de estas divisiones.

\section{Antecedentes: Características sociales relevantes de las ciencias}

En la actualidad, diferentes instancias como organismos internacionales, fundaciones o universidades (véase la bibliografía) con distintos enfoques metodológicos, realizan diagnósticos o evaluaciones sobre el desempeño de las ciencias, documentos de este tipo muestran que hay un interés vigente en aquellas y resultan muy útiles para acceder a descripciones más precisas del ámbito científico según varíe su presupuesto, temáticas u organización, entre otros aspectos, y se hallan trabajos de esta índole disponibles con enfoques de nivel nacional, regional y global. No se busca una exposición detallada de cada fuente, sino una síntesis que permita mostrar, en la brevedad de un artículo, algunas condiciones sociales relevantes de las disciplinas científicas.

Un primer apunte. A pesar de que en el período 2004-2015 las publicaciones científicas en Iberoamérica aumentaron en un $123 \% 0^{5}$, el mundo contemporáneo vive sumergido en una avasalladora saturación de información que se vale de todos los medios de comunicación y de los mismos canales que la ciencia también utiliza para divulgar el saber científico, a saber: libros, sitios en internet, televisión, exposiciones, cine, prensa radial y escrita, diccionarios, enciclopedias, antologías y un largo etcétera de opciones para difundir saberes de toda índole; por lo cual, es ingenuo presumir que la mayor cantidad de dicha información es equiparable a un mayor impacto del conocimiento, al menos por dos razones: se ignorarían las condiciones de recepción y procesamiento de información junto a las características culturales de las personas informadas, así como se descuidaría la diversidad de fuentes en la producción de información, mucha de la cual puede ser elaborada con fines de entretenimiento o propaganda, sin atenerse a ningún rigor o evidencia. Considérese además escenarios especialmente frecuentes en América Latina, en los cuales se imponen simulacros de regímenes electorales de masas $^{6}$, en donde se incuba una expectativa reducida de democracia en la cual la votación de una mayoría podría definir

\footnotetext{
4. Jean Piaget, Lógica y conocimiento científico. Naturaleza y métodos de la epistemología (Argentina: Editorial Proteo, 1970).

5. Mario Albornoz y Rodolfo Barrere, El estado de la ciencia. Principales indicadores de ciencia y tecnología iberoamericanos/interamericanos (Argentina: RICYT, OEI, 2016).

6. Helio Gallardo, Democratización y democracia en América Latina (Colombia: Ediciones Desde Abajo, 2007).
} 
lo que se acepta o no, incluyendo las hallazgos científicos, mezclando confusamente el respeto por la diversidad cultural y la libertad para decidir qué hacer con los saberes, a la par de los impedimentos socialmente producidos para discernir tipos de conocimientos y las discrepancias entre sí según su nivel de objetividad.

Muestra de la discusión anterior, es que pese a la creciente producción de información científica, la tendencia en Iberoamérica es su bajo consumo, una débil cultura científica informativa, ya que apenas se ronda una décima parte de población interesada en las divulgaciones científicas, esto significa que la ciencia y sus agentes interesados aun no llegan a difundir sus saberes al $90 \%$ de la población; ante esto, los principales motivos que se aducen en la población son la falta de tiempo o la ininteligibilidad de los conceptos o los hallazgos científicos, lo cual puede a su vez aludir a dificultades de la población para obtener las competencias para aprovechar el conocimiento científico, así como los déficits estructurales en educación ${ }^{7}$. Paradójicamente, en contraste con este desapercibimiento, la profesión de científico aún logra obtener un prestigio relativamente alto si se les compara con políticos, militares o curanderos, pues existen expectativas promisorias sobre sus alcances tentativos e incluso optimismo sobre los riesgos que asumen ${ }^{8}$; esto quiere decir que gran parte de la población da credibilidad al trabajo científico, pero no se informa de sus procesos y resultados. De tal modo, la coexistencia intermitente de acercamientos y distanciamientos de la sociedad respecto de los aportes científicos, propicia que las poblaciones acusen a la ciencia ante eventuales fallos y, a la vez, exijan su compromiso cuando se enfrentan problemas ${ }^{9}$.

En el contexto mundial, acontecimientos recientes como los accidentes en plantas nucleares, los problemas climáticos o eventos naturales, suelen socavar, en ocasiones intermitentemente, la confianza y aminorar las expectativas de la población en los alcances de la ciencia y la tecnología; pero, paradójicamente, también presionan a los gobiernos y otros actores sociales para impulsar mayores gestiones en investigación y desarrollo como medidas complementarias a la atención de tales situaciones ${ }^{10}$, los cuales generan focos de debates sobre los problemas urgentes de las ciencias, estos últimos a su vez se ven implicados en múltiples facetas de enlace entre las ciencias sociales, las políticas públicas y objetivos de otras instituciones, en donde prevalece la débil relación entre tomadores de decisiones y científicos ${ }^{11}$. Más aun, la formulación de políticas científicas no logra integrar de manera equitativa a comunidades de científicos, estados, empresas, organizaciones no lucrativas e incluso otros sectores de la sociedad civil, por lo cual se muestra un déficit democrático en la formulación de agendas científicas y tecnológicas, que le aleja

7. UNESCO, Oficina Regional de Educación para América Latina y el Caribe. Situación educativa de América Latina y el Caribe (Chile: Ediciones del Imbunche, 2013).

8. Mario Albornoz, Lourdes Arana y Álvaro Marchesi, ed, Cultura cientifica en Iberoamérica. Encuesta en grandes núcleos urbanos (España: OEI, FECYT, RICYT, 2009).

9. Queda pendiente de obtener otros tantos datos homólogos para poder definir cómo los mismos científicos se mantienen o no informados de investigaciones y resultados en disciplinas distintas de aquellas en las cuales se desempeñan, para pormenorizar sobre las relaciones también socialmente condicionadas entre científicos de distintos campos y disciplinas.

10. UNESCO Science Report: towards 2030 (Luxemburgo: UNESCO, 2015).

11. Gómez, Jusmary, «Un diálogo entre las ciencias sociales y los decisores de políticas públicas», Cuadernos del CIPS (2014): 182-189. 
de problemas sociales concretos ${ }^{12}$, justo en donde se juega buena parte de la credibilidad y apoyo a las ciencias.

En el escenario de los países periféricos, y en particular en la región de América Latina, el problema anterior de vincular ciencia y sociedad es aún más intrincado, ya que desde la década de 1990 los Estados del subcontinente han restringido o estancado sus funciones en ciencia y tecnología ${ }^{13}$, la región es más susceptible a los discursos sobre la importancia de la ciencia para el desarrollo que provienen de entidades internacionales o potencias económicas, que por tanto influyen en la agenda política. Los elementos reseñados evidencian una influencia política en las ciencias, con lo cual se trata de reconocer la presencia de luchas de poder para gestionar o controlar el conocimiento y sus aplicaciones, lo cual configura grupos de presión que pueden eventualmente atentar contra la aplicación de la investigación y sus productos como respuestas a necesidades de una región o comunidad, como es notable en América Central, en donde el paso del modelo de sustitución de importaciones al modelo aperturista hacia la competitividad de mercado en el último cuarto del siglo pasado, marcó una clara política de menor y condicionada inversión en ciencia, complaciente con los modelos exógenos de desarrollo ${ }^{14}$.

Ya a nivel global es bastante desequilibrado el vínculo entre inversión en ciencias y su rentabilidad, no todo el conocimiento sirve para fines comerciales o se replica directamente en crecimiento económico, lo cual propicia niveles de inestabilidad muy variados en el apoyo a la investigación básica según los países y las fuentes de financiamiento ${ }^{15}$. Por estas razones, entre otras, la brecha en inversión a nivel mundial en investigación y desarrollo aún señala la concentración de los recursos financieros para este fin, en Estados Unidos, por el orden del 28\%; mientras China obtiene el 20\%, la Unión Europea el 19\% y Japón el 10\%, de modo que el resto del mundo que reúne a más de dos terceras partes de la población, solo percibe poco menos de una cuarta parte de estos recursos ${ }^{16}$.

Además de las inequidades globales se muestran las regionales. En países de la Unión Europea, junto con Estados Unidos, Japón, Corea del Sur y China, la inversión en investigación y desarrollo como porcentaje del PIB promedia un $2,4 \%{ }^{17}$, mientras que en América Central el promedio de inversión en ciencias está por debajo del $0,5 \%{ }^{18}$. En términos ya no relativos, sino proporcionales, la diferencia es todavía mayor al considerar el presupuesto de cada país; para muestra de esto, en el 2016, por cada $\$ 83,3$ en Chile y $\$ 90,1$ en México que se invierten per cápita en investigación y desarrollo, en Corea del Sur se ponen $\$ 1548,5$ y en Israel $\$ 1584,4^{19}$.

12. Ronny Viales, «Los elementos básicos para la formulación de políticas científicas, tecnológicas y de innovación para la cohesión social. Una visión CTS», Revista de Ciencias Sociales, n. ${ }^{\circ} 153$ (2016): 101-120.

13. Ibíd.

14. Viviana Guerrero, «Aproximación teórica e histórica para el análisis de las políticas científicas desde el enfoque CTS en América Central, 1980-2014», Revista de Ciencias Sociales, n. ${ }^{\circ} 153$ (2016): 87-100.

15. UNESCO Science Report, 2015.

16. Ibid.

17. OECD, Main Science and Technology Indicators, acceso: 24 de abril de 2018, http://www.oecd.org/sti/msti.htm

18. Aproximación teórica e histórica, 87-100.

19. Main Science and Technology Indicators. 
La colocación de la inversión en América Latina para investigación se orienta predominantemente en ciencias aplicadas, en donde se presumen más redituables los recursos en términos de crecimiento económico; así esta inversión en diez años (del 2005 al 2014) aumentó en un 107\% y se concentra en Brasil, México y Argentina en un $91 \%{ }^{20}$; lo cual muestra que por razones sociales la ciencia puede aumentar o estancar su involucramiento social y disponibilidad de recursos, como se evidencia en el período señalado, aunque lo hace desigualmente en condiciones predominantemente controladas por lógicas de mercado, en donde la organización capitalista globalizada de las economías científicas ofrece casos interesantes que ameritarían una inspección minuciosa, como la inversión en investigación farmacéutica, en genética y biotecnología; allí se elevan preguntas fundamentadas sobre quiénes financian grandes presupuestos de investigación, cuáles condiciones imponen sobre los objetos y métodos de pesquisar ${ }^{21}$ o sobre cómo se produce valor y renta del conocimiento por vía de la innovación tecnológica, las patentes o restricción de divulgación de conocimientos; por tanto, se cuestiona quiénes se benefician o se perjudican con ciertas prácticas científicas.

Por si fuera poco, no se pueden tomar aisladamente los montos invertidos en investigación y desarrollo, a lo largo de la historia reciente el desarrollo tecnológico ha estado asociado muy directamente con las campañas bélicas y el comercio de guerra; pues en potencias económicas y países altamente industrializados, una parte grande de estos presupuestos se orienta hacia la industria militar aunque su impacto económico no es decisivo $^{22}$. Otra tendencia importante por considerar es que los países con alto desarrollo industrial y alta inversión en investigación y desarrollo, reciben sus recursos cada vez en mayor medida del sector empresarial y con menor cuota del Estado, lo cual reconfigura las relaciones sociales y demandas para con las comunidades de científicos; aquí los casos más sobresalientes son los de Japón, China y Corea del Sur, donde más del 75\% de sus fondos para investigación proviene del sector privado ${ }^{23}$.

Los problemas, sin embargo, no están solo en las audiencias tentativas de la ciencia o en el financiamiento, hay secuelas también de la inequidad en sus productores. Hoy la ciencia y la tecnología generalizadamente tienen una cantidad de profesionales que supera por más del doble la fuerza laboral empleada a fines del siglo XX, eso suma hoy un aproximado global de 7,8 millones de científicos e ingenieros empleados en investigación, con una mayoría del 22\% ubicada en la Unión Europea; mientras menos de una vigésima parte corresponde a América Latina y el Caribe ${ }^{24}$. Los recursos no son solo cifras dinerarias, se expresan también en la fuerza de trabajo disponible; en la Unión Europea, para el 2016, existe un equivalente a más de 1888000 investigadores de tiempo completo,

20. Albornoz, Mario y Rodolfo Barrere, ed., El estado de la ciencia. Principales indicadores de ciencia y tecnología iberoamericanos / interamericanos (Argentina: RICYT, OEI, 2016).

21. Werner, Klaus y Hans Weiss, El libro negro de las marcas: el lado oscuro de las empresas globales (México: DeBolsillo, 2006).

22. Dunne, Paul y Derek Braddon. Economic impact of military research and development (Bélgica: Flemish Peace Institute, 2008).

23. Main Science and Technology Indicators.

24. UNESCO Science Report, 2015. 
en China es de aproximadamente 1692000 y en Estados Unidos es de más de 1379000, mientras en México ronda los 30000 y en Chile los $9000^{25,26}$.

\section{Discusión: Contra la ignorancia científica de la ciencia}

En conjunto, los hallazgos anteriores son triplemente relevantes para sembrar el argumento central. Por un lado muestran que las ciencias aplicadas, naturales y demás, están directamente afectadas por sus condiciones sociales; por otra parte, todos estos datos sobre las condiciones de las ciencias naturales provienen de grupos de investigación de ciencias sociales, como también se aprecia en estudios de escala global ${ }^{27}$; es decir, son solo una muestra de los tipos de beneficios que las ciencias sociales también le pueden reportar a las ciencias naturales, aportándoles conocimiento fundamentado y de utilidad estratégica para su orientación; pero, paralelamente existen también algunas fracciones en las academias de ciencias sociales o las humanidades que atacan infundadamente a las ciencias naturales ${ }^{28}$, mientras en otros lares de las ciencias sociales sí logran desplegar crítica e investigación fundamentada sobre las ciencias naturales, o incluso ponen en evidencia la colaboración que también las ciencias sociales pueden hacer a las ciencias naturales y viceversa ${ }^{29}$. Finalmente, con un sentido algo extraño, estos estudios en las perspectivas mundial, regional y nacional que hacen las ciencias sociales sobre las circunstancias de las ciencias, se enfocan predominantemente en las ciencias naturales; es decir, son investigaciones de ciencias sociales que se excluyen a sí mismas del dominio de preocupaciones científicas, esto es, no se consideran como parte del objeto de estudio, sin dar justificación suficiente se enfocan en ciencias naturales y tecnología, descuidando la otra mitad del gremio: ciencias sociales e intervención social; este es un vacío encontrado.

No se vaya a inadvertir que, aun incorporando a las ciencias sociales como parte del objeto de estos diagnósticos, se podría superar la incongruencia anterior solo para topar con las dificultades epistemológicas de tener que ser a la vez sujeto y objeto de investigación, lo cual explica por qué las ciencias sociales tienden a ser más complejas y problemáticas que las ciencias naturales, pero nunca por eso inservibles ${ }^{30}$. Al respecto, si bien aún no se identifican balances sobre la investigación en ciencias sociales, sí existe trecho avanzado en una importante discusión teórica e histórica sobre los impactos de las ciencias sociales y sus relaciones con los problemas sociales ${ }^{31}$, así como la espinosa relación

25. Main Science and Technology Indicators.

26. Hay también implicaciones locales, en Costa Rica por ejemplo, la producción científica depende de un reducido número de actores estratégicos con dificultades para incorporar suficientes mujeres y hacer relevo generacional. Estado de la ciencia, la tecnología y la innovación (Costa Rica: Programa Estado de la Nación, 2014). De modo que la desigualdad en las ciencias suma otras tantas dificultades para integrarse a la sociedad considerando criterios de género y edad, especialmente agudos en América Central. Guerrero, Viviana, «Aproximación teórica e histórica para el análisis de las políticas científicas desde el enfoque CTS en América Central, 1980-2014», Revista de Ciencias Sociales, n. ${ }^{\circ} 153$ (2016): 87-100.

27. UNESCO Science Report, 2015.

28. Koertge, Noretta, A house built on sand. Exposing posmodernist myths about science (Estados Unidos: Oxford University Press, 1998).

29. Piaget, Jean, Tratado de lógica y conocimiento científico. Volumen VI. Epistemología de las ciencias del hombre (Argentina: Editorial Paidós, 1979).

30. Norbert Elias, Compromiso y distanciamiento (España: Ediciones Península, 2002).

31. Raymond Aron, Estudios sociológicos (Calpe, España: Editorial Espasa, 1989). 
entre investigación social e intervención social ${ }^{32}$. Hasta aquí, la evidencia anterior, aunque solamente es parcial, converge en la tesis de que la ciencia requiere respaldo político, económico y cultural; por tanto, nunca se centra únicamente en la validez, veracidad o innovación de sus resultados, estos varían según cuánto y cómo se publica, quiénes se informan, cuáles investigaciones se financian y en dónde, qué imaginarios se tienen sobre los científicos y cómo se vinculan estos con otros actores sociales, entre otras condiciones. El rigor empírico, metódico y teórico para lograr ciencias de impacto es imprescindible pero no autosuficiente.

Si se considera que buena parte de la dinámica del desarrollo del conocimiento científico se debe a la reciprocidad entre conocer e ignorar ${ }^{33}$, esto se puede traducir de manera quizá más precisa como una ignorancia conocida, toda vez que se tiene mayor precisión sobre lo que es desconocido pudiendo no serlo y, por tanto, definir qué se sabe con claridad de las sociedades actuales, para a partir de esto establecer qué se ignora para indagarlo; brinda el vector de la investigación en las ciencias sociales y, en este caso específico, la interrogación de las ciencias sociales se puede convertir en un programa de investigación también.

Incluso, consintiendo el nivel mayor de complejidad alcanzado teórica, metódica y empíricamente durante varias décadas por las ciencias sociales ${ }^{34}$, estas no siempre pueden reclamar novedad, habrá que reconocer también que mucha investigación ejecutada desde ámbitos propios de las ciencias sociales solo actualiza datos, replica hallazgos previos o, en el peor de los casos, puede incurrir en el discurso críptico para presumir algún grado de profundización en realidad trivial o del todo absurdo ${ }^{35}$ o hasta generar mezclas absurdas entre ciencias sociales y ciencias naturales ${ }^{36}$; incluso, sesgar ideológicamente procesos de investigación de modo que la objetividad sea solo fingida, para ocultar tendencias políticas ${ }^{37}$ o para encubrir ventajas para clases sociales dominantes ${ }^{38}$. En suma, las ciencias sociales no pueden ser exoneradas de cuestionamientos sobre sus aportes reales justamente porque también están enrevesadas en todas sus condiciones sociales de producción y uso. La situación actual es lo suficientemente compleja para permitir la coexistencia, a veces conflictiva, a veces inadvertida, de ciencia de impacto en la toma de decisiones ${ }^{39}$ y ciencia prosélita por intereses espurios ${ }^{40}$, con variable margen de calidad o mediocridad.

32. Orlando Fals-Borda, Una sociología sentipensante para América Latina (Colombia: Editorial Siglo del Hombre-CLACSO, 2009).

33. Karl Popper, La lógica de la investigación científica (España: Editorial Tecnos, 1980).

34. George Ritzer, ed, Encyclopedia of Social Theory (Estados Unidos: Sage Publications, 2005).

35. Stanislav Andreski, Las ciencias sociales como forma de brujería (España: Taurus Ediciones, 1973).

36. Bricmont Jean y Alan Sokal, Imposturas intelectuales (España: Editorial Paidós, 1999).

37. Ralph Miliband, El estado en la sociedad capitalista (México: Siglo XXI Editores, 1978).

38. Jürgen Habermas, Ciencia y técnica como «ideología» (España: Editorial Tecnos, 1968).

39. Bárbara García, «Producción de conocimiento científico y toma de decisiones en el campo de la política pública. Modelos, tensiones y perspectivas», Revista Debate Público. Reflexión de Trabajo Social, año 4, n. ${ }^{\circ} 8$ (2014): 51-60.

40. Michel Chossudovsky, «Dependencia y transferencia de tecnología intelectual», Revista Nueva Sociedad, n. $^{\circ} 25$ (1976): 95-101. 
Por su parte, las ciencias sociales construyen también otros muchos objetos de estudio a partir de individuos y grupos con márgenes condicionados de autogobierno, con carencias y capacidades, en contexto social, entendiendo que los científicos sociales son parte del objeto. Tal como se mostró antes, se trata de sujetos cuyas condiciones sociales varían según su área de trabajo, su país, su género o edad, su acceso a recursos, y por tanto tales condiciones reverberan sobre sus posibilidades de acción. Por otra parte, el conocimiento predominantemente intuitivo que las personas tienen de su contexto, genera con frecuencia que las ciencias sociales vayan a cuestionar el sentido común así como las prácticas consuetudinarias ${ }^{41}$ que también afectan a los científicos; nadie es un científico puro durante toda su vida ${ }^{42}$, gran parte del tiempo en la vida de una persona científica se consume en atender asuntos de convivencia y sociabilidad, sin percatarse necesariamente de esto; por lo tanto, resulta quizá difusa, pero resueltamente frecuente, la incidencia de las relaciones humanas, pero esto no desacredita necesariamente el potencial de las ciencias sociales para discernir con mayores rigores las causas y efectos múltiples de las relaciones sociales, ni tampoco permite presumir que los individuos quienes no son científicos sociales sean ignorantes plenos de su sociedad; se trata en realidad de un punto de tensión entre lo sabido y lo desconocido de lo social, por ello la verdad objetiva de lo social resulta las más de las veces conflictiva, pues puede llegar a confrontar ignorancias, revelar prejuicios o desacreditar creencias.

\section{Consideraciones finales: La ciencia de la ciencia para discernir los propósitos de la investigación}

La ciencia ya ha avanzado, como se mostró en los antecedentes, en la investigación de sí misma, es decir, la incursión en la ciencia de la ciencia no tarda en señalar que los factores sociales la hacen posible o la constriñen. Consecuentemente el impacto de las ciencias puede variar según cómo se gestionan no solo los procesos de investigación, sino también su divulgación y debate para aplicarlo; por eso, no deja de tener cabida un llamado de modestia, el acervo de conocimiento en ciencias sociales insiste en que los principales conflictos sociales son socialmente producidos, así como lo son las alternativas y soluciones a los problemas sociales. Sabiendo que el impacto del conocimiento científico requiere establecer relaciones de trabajo colaborativo con otras instancias sociales ${ }^{43}$, ninguna ciencia, al ser solo una parte de la sociedad, puede ser considerada como la panacea de lo social, por ende tener conocimiento mayor sobre lo social solo aporta parcialmente, pero no garantiza el mejoramiento ni el empeoramiento de una situación, muchos otros factores intervienen en estos resultados.

Las ciencias sociales y los conocimientos que estas producen pueden o no resultar de provecho. En ocasiones los aportes de las ciencias sociales no son convenientes para los intereses que defienden algunos sectores o grupos, especialmente los hegemónicos, cuando ponen en evidencia desigualdades, relaciones de violencia o explotación; o pudiese

41. Pierre Bourdieu, Jean-Claude Passeron y Jean-Claude Chamboredon, El oficio de sociólogo (México: Siglo XXI Editores, 2007).

42. Bertrand Russell, La perspectiva cientifica (España: Editorial Ariel, 1981).

43. Viales, «Los elementos básicos para la formulación de políticas científicas, tecnológicas y de innovación para la cohesión social», 101-120. 
ser también que los aportes e incluso los errores científico sociales, resulten perjudiciales para poblaciones marginadas o empobrecidas, según el uso instrumental o tergiversación que se les dé para manipular o estigmatizar a estos sectores.

Las ciencias sociales, entendidas críticamente, como productoras condicionadas de conocimientos objetivos sobre lo social, no se pueden concebir como disciplinas irrevocablemente destinadas a la consecución del orden social, primero por reconocer sus márgenes de error, y segundo, porque incluso siendo acertados los conocimientos disponibles, aplicarlos en situaciones específicas requiere múltiples consideraciones casuísticas, esto es necesario para evitar una reducción de la ciencia social a técnica social conservadora del status quo ante.

En el otro extremo, acechan concepciones mesiánicas de las ciencias sociales, que proclaman a estas disciplinas como las protagonistas de la transformación civilizatoria y reclaman para sí la tarea de la emancipación humana; esto es históricamente inconsecuente con el hecho de que las dinámicas de opresión y emancipación en los seres humanos es anterior a la existencia de las ciencias modernas y, este mesianismo, puede degenerar en visiones idílicas o deformadas de las poblaciones marginadas, explotadas, agredidas o asesinadas que no tendrían ninguna responsabilidad ni capacidad de agencia sobre sus condiciones de vida, aunque evidentemente el nivel de impacto de cada una de las partes sociales varía enormemente, por cuanto no es la misma participación histórica la que tiene un individuo que una institución milenaria. La ciencia en general exige un alto compromiso con el rigor investigativo pero no garantiza ningún tipo de redención por vía del conocimiento ${ }^{44}$.

En la ciencia también se aprende a contrariar las propias creencias, ser científico supone entender que el equívoco es parte diaria de la investigación, que los resultados en ocasiones muestran errores ${ }^{45}$, no solo aciertos; por si fuera poco, es negligente presumir una tendencia armónica entre saberes, según la cual, todo nuevo dato, simplemente se suma a la base anterior; hay limitaciones y contradicciones, las refutaciones y rectificaciones han estado a la orden del día en la historia de todas las disciplinas y esto puede generar caos o desconcierto en el conocimiento ${ }^{46}$, de tal modo la arrogancia del saber absoluto no tiene cabida en la ciencia sin asumir una auto descalificación. Por el contrario, la enseñanza científica es capaz de inculcarse dentro de un esquema polímata, bajo el cual el rigor investigativo no riñe con la pluralidad de disciplinas, objetos, teorías, métodos, técnicas, fuentes y datos; pero aunado recurrentemente al auxilio del fundamento epistemológico para evitar el relativismo y la anarquía cognoscitiva que propician que múltiples formas de ignorancia o prejuicio social se instauren como verdaderas o insondables para el entendimiento humano, con un creciente costo humano manifiesto en la forma de explotación, agresión o muerte que se presumen luego como eclosiones únicamente fortuitas, ignorando escenarios estocásticos, desdibujando toda comprensión.

Reconociendo que el saber científico social puede aportar al discernimiento de la producción social de carencias y conflictos, así como de recursos y soluciones, hoy ya

44. Max Weber, El político y el cientifico (España: Alianza Editorial, 1993).

45. Gastón Bachelard, La formación del espiritu cientifico (México: Siglo XXI Editores, 2000).

46. Alexandre Koyré, Del mundo cerrado al universo infinito (México: Siglo XXI Editores, 2015). 
existen esfuerzos por reunir distintas producciones de ciencias sociales en diversas latitudes del mundo, como lo hace el Informe Mundial de Ciencias Sociales, el ejemplar más reciente del año 2016, enfocado en el tema vertebral de la desigualdad, justamente señalando que una de las facetas de esta temática se encuentra en el acceso y producción de conocimiento sobre esa misma desigualdad, así como en las variaciones en la calidad de las mediciones en distintos rubros según país o región; es decir, no todos los países tienen el mismo conocimiento de sus condiciones sociales, unos tienen mejor y mayor información, otros tienen datos imprecisos e incluso vacíos en su documentación, esto a pesar de la tendencia global de aumento de publicaciones sobre esta temática, la cual convoca cada vez más a científicos no solo del ámbito social, sino también de ciencias de la salud ${ }^{47}$.

Las ciencias sociales tienen como tarea compartida no solo el acopio y elaboración de conocimientos, sino que la empresa científica está intrínsecamente ligada con una tarea educativa para enseñar y aprender constantemente a conocer científicamente el universo. La enseñanza de la ciencia supone la conquista y también la superación de saberes, no el apego tradicionalista o dogmático a una certidumbre, eso implica que la educación superior en universidades y otros espacios, en donde se define mucho de lo que estarán llamadas a ejercer las profesiones científicas, es interpelada por su capacidad para lograr una educación más científica y también una ciencia más educativa, calando en la producción, difusión y problematización del conocimiento, así como en la producción de vínculos con sectores sociales que se movilizan, luchan o dan testimonio de las secuelas del conflicto y la desigualdad social.

La ciencia es un saber diferente de otras experiencias humanas, ha logrado aprender y enseñar, no sin dificultades ni reveses, a distanciarse del pensamiento autoritario y, por el contrario, muestra el acecho constante de mitos, prejuicios y errores a los cuales se recurre para explicar la existencia; sin embargo, a pesar de sus vertebrales aportes para la civilización humana, no hay cabida en ninguna disciplina científica para la autocomplacencia en presunta neutralidad valorativa o superioridad moral.

El sueño positivista por excelencia del control total de la sociedad por la ciencia únicamente es posible creyendo excesivamente aminoradas las siempre mayores fuerzas de la división social del trabajo, la organización de la vida espiritual por parte de aparatos clericales, la información masiva de los medios de comunicación hegemónicos, los arbitrios culturales implementados por sistemas escolares, los estados o los mercados, entre tantos otros campos sociales, con una capacidad de influencia mucho más potente sobre representaciones colectivas y conductas singulares, sobre actores y sujetos. A contrapelo del mito que define la modernidad como la era de la ciencia, todas las disciplinas de este ámbito aun enfrentan, con desiguales recursos y cualidades heterogéneas, escenarios distintos, en donde pueden ser presas de apologías desorbitadas e instrumentalización, o quedar arrinconadas por el desinterés, la ignorancia o la tergiversación y la voraz censura.

47. ISSC, IDS, UNESCO, World Social Science Report 2016. Challenging Inequalities: Pathways to a Just World (Francia: UNESCO, 2016). 
Aspirar a conocer el universo con fundamento teórico y precisión empírica supone una confrontación con otras formas sociales del conocimiento, entabla relaciones con los usos y creencias alrededor del saber, entre los cuales existe la prohibición o descalificación de todo lo que se presente como científico; por tanto, en todas las ciencias, el propósito de investigar es siempre simultáneamente atravesado por intereses científicos, culturales, históricos y económicos, es un fin en tanto pretende conquistar verdades objetivas y también es un medio en tanto se presta para otros usos y aplicaciones, esto coadyuva dificultades insoslayables como las mencionadas antes, por esto las reflexiones fundamentadas y críticas permiten mantener vigilancia sobre las condiciones que afectan a las ciencias y sus alcances históricos, siempre en devenir, y los modos de superar o sumergirse más profundamente en la ignorancia social de la ciencia y en la ignorancia científica de lo social.

\title{
SUMMARY
}

\section{Questioning the purpose of social sciences}

\begin{abstract}
This article presents several data on the contexts of science at the regional and global levels. This data is later used to foster a discussion on the decisive effect of social conditions on scientific disciplines that imply internal inequality. This work is supported by recent documental resources and information as well as a critical theoretical position regarding science; this position affirms that society constantly defines the function and usefulness of social sciences thus pointing to the mutual learning that takes place between social and natural sciences.
\end{abstract}

Key words: science, social sciences, knowledge, inequality.

\section{RÉSUMÉ}

\section{Questioner le but des sciences sociales}

Cet article présent plusieurs données sur le contexte des sciences à niveau global et régional qui permettent une discusion ultérieure sur l'effect décisif des constions sociales des disciplines scientiques qu'impliquent des inégalités internes. Ce travail part des sources documentaires et d'information récente et pertinente liées à un positionnement teorique et critique de la science qui soutient que la société define constamment la fonction et l'utilité des sciences sociales, en soulignant l'apprentissage mutuel entre les sciences sociales et les sciences naturelles.

Mots-clés: science, sciences sociales, connaissance, inégalité.

\section{Bibliografía}

Albornoz, Mario y Rodolfo Barrere, ed. El estado de la ciencia. Principales indicadores de ciencia y tecnología iberoamericanos / interamericanos. Argentina: RICYT, OEI, 2016.

Albornoz, Mario, Lourdes Arana y Álvaro Marchesi, ed. Cultura científica en Iberoamérica. Encuesta en grandes núcleos urbanos. España: OEI, FECYT, RICYT, 2009.

Andreski, Stanislav. Las ciencias sociales como forma de brujería. España: Taurus Ediciones, 1973.

Aron, Raymond. Estudios sociológicos. Calpe, España: Editorial Espasa, 1989. 
Bachelard, Gastón. La formación del espíritu científico. México: Siglo XXI Editores, 2000.

Bourdieu, Pierre, Jean-Claude Passeron y Jean-Claude Chamboredon. El oficio de sociólogo. México: Siglo XXI Editores, 2007.

Bricmont, Jean y Alan Sokal. Imposturas intelectuales. España: Editorial Paidós, 1999.

Chossudovsky, Michel. «Dependencia y transferencia de tecnología intelectual». Revista Nueva Sociedad, n. ${ }^{\circ}$ 25, Colombia: Fundación Friedrich Ebert (1976): 95-101.

Dunne, Paul y Derek Braddon. Economic impact of military research and development. Bélgica: Flemish Peace Institute, 2008.

Elias, Norbert. Compromiso y distanciamiento. España: Ediciones Península, 2002.

Fals-Borda, Orlando. Una sociología sentipensante para América Latina. Colombia: Editorial Siglo del Hombre-CLACSO, 2009.

Gallardo, Helio. Democratización y democracia en América Latina. Colombia: Ediciones Desde Abajo, 2007.

García, Bárbara. «Producción de conocimiento científico y toma de decisiones en el campo de la política pública. Modelos, tensiones y perspectivas». Revista Debate Público. Reflexión de Trabajo Social, año 4, n. ${ }^{\circ} 8$, Universidad de Buenos Aires (2014): 51-60.

Gómez, Jusmary. «Un diálogo entre las ciencias sociales y los decisores de políticas públicas». Cuadernos del CIPS. Experiencias de investigación social en Cuba. Centro de Investigaciones Psicológicas y Sociológicas, Cuba, UNICEF (2014): 182-189.

Guerrero, Viviana. «Aproximación teórica e histórica para el análisis de las políticas científicas desde el enfoque CTS en América Central, 1980-2014». Revista de Ciencias Sociales, n. ${ }^{\circ}$ 153, Universidad de Costa Rica (2016): 87-100.

Habermas, Jürgen. Ciencia y técnica como «ideología». España: Editorial Tecnos, 1968.

ISSC, IDS, UNESCO. World Social Science Report 2016. Challenging Inequalities: Pathways to a Just World. Francia: UNESCO, 2016.

Koertge, Noretta. A house built on sand. Exposing posmodernist myths about science. Estados Unidos: Oxford University Press, 1998.

Koyré, Alexandre. Del mundo cerrado al universo infinito. México: Siglo XXI Editores, 2015.

Mandel, Ernest. Capitalismo tardio. México: Ediciones ERA, 1979.

Miliband, Ralph. El estado en la sociedad capitalista. México: Siglo XXI Editores, 1978.

OECD. Main Science and Technology Indicators. Acceso: 24 de abril de 2018. http://www.oecd.org/sti/msti.htm

Piaget, Jean. Lógica y conocimiento científico. Naturaleza y métodos de la epistemología. Argentina: Editorial Proteo, 1970.

Piaget, Jean. Tratado de lógica y conocimiento científico. Volumen VI. Epistemología de las ciencias del hombre. Argentina: Editorial Paidós, 1979.

Popper, Karl. La lógica de la investigación científica. España: Editorial Tecnos, 1980.

Programa Estado de la Nación. Estado de la ciencia, la tecnología y la innovación. Costa Rica: Programa Estado de la Nación, 2014.

UNESCO. UNESCO Science Report: towards 2030. Luxemburgo: UNESCO, 2015. 
UNESCO, Oficina Regional de Educación para América Latina y el Caribe. Situación educativa de América Latina y el Caribe. Chile: UNESCO, Ediciones del Imbunche, 2013.

Ritzer, George, ed. Encyclopedia of Social Theory. Estados Unidos: Sage Publications, 2005.

Russell, Bertrand. La perspectiva científica. España: Editorial Ariel, 1981.

Viales, Ronny. «Los elementos básicos para la formulación de políticas científicas, tecnológicas y de innovación para la cohesión social. Una visión CTS». Revista de Ciencias Sociales, n. ${ }^{\circ}$ 153, Universidad de Costa Rica (2016): 101-120.

Weber, Max. El político y el científico. España: Alianza Editorial, 1993.

Werner, Klaus y Hans Weiss. El libro negro de las marcas: el lado oscuro de las empresas globales. México: Editorial DeBolsillo, 2006. 